\title{
Propagation of nuclear data uncertainty: Exact or with covariances
}

\author{
D. Rochman a , A.J. Koning, S.C. van der Marck, A. Hogenbirk, and D. van Veen
}

Nuclear Research and Consultancy Group (NRG), Petten, The Netherlands

\begin{abstract}
Two distinct methods of propagation for basic nuclear data uncertainties to large scale systems will be presented and compared. The "Total Monte Carlo" method is using a statistical ensemble of nuclear data libraries randomly generated by means of a Monte Carlo approach with the TALYS system. These libraries are then directly used in a large number of reactor calculations (for instance with MCNP) after which the exact probability distribution for the reactor parameter is obtained. The second method makes use of available covariance files and can be done in a single reactor calculation (by using the perturbation method). In this exercise, both methods are using consistent sets of data files, which implies that covariance files used in the second method are directly obtained from the randomly generated nuclear data libraries from the first method. This is a unique and straightforward comparison allowing to directly apprehend advantages and drawbacks of each method. Comparisons for different reactions and criticality-safety benchmarks from ${ }^{19} \mathrm{~F}$ to actinides will be presented. We can thus conclude whether current methods for using covariance data are good enough or not.
\end{abstract}

\section{Introduction}

Since the beginning of the century, the nuclear data evaluation community is putting more and more attention to the assessment of uncertainties. This increased interest concerns both basic data (cross sections, emission spectra...) and calculated quantities for large systems, such as neutron multiplication factor $\left(\mathrm{k}_{\mathrm{eff}}\right)$ for a reactor, void coefficient, leakage flux and others. With the larger availability of covariance files, as in the ENDF/B-VII.0 library [1] or in the TENDL-2008 and TENDL-2009 libraries [2,3], more and more studies are using this information to deduce target accuracies for future reactors [4] and therefore future priorities for experimental measurements of differential data. As of today, two methods can be used to propagate uncertainties from nuclear data to quantities of large scale systems. The first one, the perturbation method associated with covariance files (see for instance Ref. [4]) is the most used method among the reactor physics community. Both sensitivity profiles and covariance data need to be combined in order to obtain final uncertainties. At NRG, a method has been developed to generalize the perturbation card of the MCNP code to obtain sensitivity matrices as a function of isotope and energy bin [5]. This sensitivity profile is then combined with the available covariance information to obtain an uncertainty on a $\mathrm{k}_{\mathrm{eff}}$.

More recently, thanks to the huge increase in computer power of these last decades, a new method has been developed and applied at NRG, based on Monte Carlo calculations and called "Total Monte Carlo", or TMC (see Refs. [6-8]). This latest method relies on a large number of calculations, all alike but with unique nuclear data in each of them. The result is a probability distribution from which different moments can be extracted.

\footnotetext{
a e-mail: rochman@nrg.eu
} permits unrestricted use, distribution, and reproduction in any noncommercial medium, provided the original work is properly cited. 


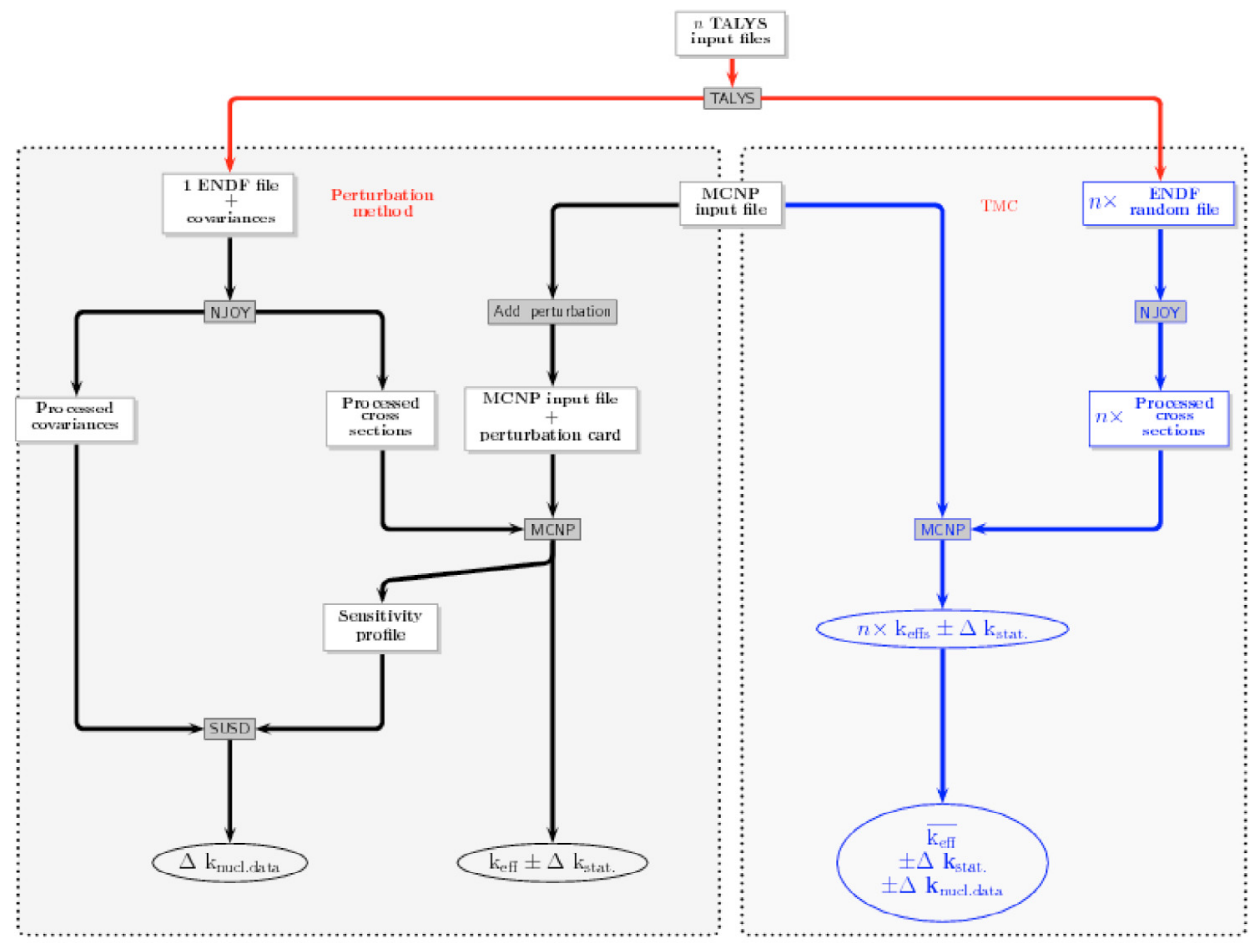

Fig. 1. Flowchart of the uncertainty propagation for the TMC and perturbation methods.

As long as only one method existed, it was not possible to compare it with another one, and results were accepted as is because of no alternative approach. Nowadays, with two different methods to propagate uncertainties, it is in principle possible to compare the calculated uncertainties for each method.

In this study, efforts are first invested to guarantee that both methods start from the same quantities (same nuclear data and same covariance information). This is achieved by creating together inputs for the perturbation method and the TMC method. Then careful processing steps are carried out for each method, making sure again that the same system of calculation is applied (NJOY processing, MCNP geometry input and nuclear data libraries). In this paper, details of the procedure are presented for a few criticality-safety benchmarks. Results for the $\mathrm{k}_{\mathrm{eff}}$ quantity are presented and advantages and drawbacks of both methods are given.

\section{Methodology}

The selected systems for the comparison of the methods are a series of criticality-safety benchmarks from the ICSBEP database [9]. In the following, six benchmarks are chosen ranging from thermal to fast systems. These benchmarks were already extensively studied for the validation of the ENDF/BVII.0 library in Ref. [10]. For each of the selected benchmarks, the impact of the nuclear data uncertainty for a single isotope is studied. The selected isotopes range from ${ }^{19} \mathrm{~F}$ to ${ }^{240} \mathrm{Pu}$, to cover as much as possible the mass range of important isotopes ${ }^{1}$. Each isotope information (cross sections, differential data, covariances) are processed either in pointwise data (for $k_{\text {eff }}$ calculations) or in 33 energy groups for the perturbation method. The global flowchart of steps for each methods is presented in Fig. 1 .

\footnotetext{
${ }^{1}$ Light elements, such as hydrogen or oxygen are also of crucial importance, but can not be easily modeled in terms of nuclear reactions.
} 
The same MCNP model (with MCNP version 4C3 [11]) for each of the selected criticality-safety benchmarks is used in both the TMC and perturbation methods. On the same manner, the same version of the processing tools NJOY [12] (version 99.259), PUFF [14] (version IV 6.1.0) and SUSD [15] are used for the entire study.

In order to compare results from the two methods, the starting points have to be similar. A difficult and necessary step is to obtain equivalent nuclear data files to be used by both methods. On one side, the TMC method is using a large number of random ENDF files, and on the other side, the perturbation method is using a single ENDF file containing covariance information. It has to be guaranteed that the cross sections from the single ENDF file used in the perturbation approach is the average of the large number of random files. Additionally, the covariance information (uncertainties and correlations) has to represent the set of random ENDF files used in the TMC approach. To ensure this, the technique applied in this study is to generate these ENDF files from the same set of runs using the TALYS code (version $1.2[16]$ ).

The procedure to generate random ENDF files together with an ENDF file containing the average cross sections and the covariance information was detailed in Ref. [6]. In summary, 20 to 30 theoretical parameters are all varied together within pre-determined ranges to create TALYS inputs. With the addition of a large number of random resonance parameters, nuclear reactions from thermal energy up to $20 \mathrm{MeV}$ are covered. The TALYS system creates random ENDF nuclear data files based on these random inputs. At the end of the random file generation, the covariance information (average, uncertainties and correlations) are extracted and formatted into an ENDF file. This method allows to cover the top part of Fig. 1, from the " $n$ TALYS input files", to the "1 ENDF file + covariances" and " $n \times$ ENDF random files".

\subsection{Codes and programs}

In this study, a few codes and programs are used. Depending on which method is used to propagate uncertainties, different nuclear related programs have to be installed, checked and connected. These codes are: common to TMC and Perturbation method: TALYS system, NJOY (ACE), MCNP; for the TMC method: None; and for the Perturbation method: NJOY (ERRORR) or PUFF, an "Add perturbation" module and SUSD. The TALYS system, version 1.2, is a nuclear reaction codes package [16] used by both basic nuclear physics and applied nuclear science groups from all over the world. NJOY, version 99.259, is a modular code for nuclear data processing $[12,13]$. To produce files used by MCNP, the ACER module is needed and the ERRORR module is used to process covariance files.

MCNP, version 4C3, is the well-known MCNP general-purpose Monte Carlo N-Particle code that can be used for neutron, photon, electron, or coupled neutron/photon/electron transport [11]. In the present usage, benchmark descriptions are obtained from the ICSBEP list and are used by MCNP.

PUFF, version IV 6.1.0, is used to process resonance parameter covariance information and pointwise covariance matrices into group-averaged covariances matrices on a user-supplied group structure [14]. It can be used instead of the ERRORR module.

SUSD calculates standard deviation given cross-section covariance matrices and sensitivity profiles [15].

Finally, the addition of perturbation cards to a MCNP input is automated.

\subsection{Perturbation approach}

The perturbation approach relies in principle on a unique "NJOY+MCNP+SUSD" calculation. The inputs are the geometry MCNP input file (common to the TMC approach) and the ENDF file containing covariances (consistent with the $n$ ENDF files used in TMC).

As shown in Fig. 1, the ENDF file is processed by NJOY to produce processed cross sections (used by MCNP) and processed covariances (used by SUSD). In the following, uncertainties are to be interpreted as standard deviations, unless otherwise stated. 
The "Add perturbation", "MCNP input file + perturbation card" and "MCNP" boxes in Fig. 1 presents the essential part of the sensitivity calculation. It is based on the most commonly used radiation transport code MCNP [11]. The sensitivity profile $S$ is defined as the relative change in a response parameter $R$ due to a relative change in a cross section in a particular energy group $g S=\frac{(\delta R) / R}{\left(\delta \sigma_{q}\right) / \sigma_{q}}$. In this case, the response parameter is a scalar quantity, which is a function of the incident neutron energy. The sensitivity profile $S$ is obtained using the perturbation option of MCNP, which corresponds to the "PERT"-card:

1. A cross section is selected for which the profile is to be generated. In the following, four cross sections will be considered: elastic, inelastic, fission and capture cross sections. Only one specific isotope is varied each time.

2. A material card is created in which the atomic density for the relevant isotope is increased by $1 \%$.

3. a "PERT"-card is created specifying that the relevant material is replaced by the perturbed material in each if the cells in which the material is present. Perturbation cards are given for all energy groups. In this paper, the 33-energy group structure (from thermal energy to $20 \mathrm{MeV}$ ) is adopted.

4. Finally, MCNP is run with these modifications in the input. In the MCNP output, a table is given with the results of the perturbations with statistical uncertainties and, in case of criticality benchmarks, a $\mathrm{k}_{\mathrm{eff}}$ values with statistical uncertainties.

This method is then applied to criticality benchmarks as defined in the ICSBEP list [9]. This part is represented in Fig. 1 by the "SUSD" box. The sensitivity results and the processed covariances are combined together with the SUSD code, in a similar energy group. Sensitivities are calculated for cross sections only (resonance region and fast neutron range). Thus, the effect of angular distribution, double differential data and, in the case of actinides nu-bar and fission neutron spectrum, can not be included in this approach. The calculated quantity is an uncertainty on $\mathrm{k}_{\mathrm{eff}}$ due to nuclear data.

\subsection{Total Monte Carlo}

The "Total Monte Carlo" method for nuclear data uncertainty propagation was presented in Ref. [6] and extensively applied to criticality-safety benchmarks [7], void coefficient of a Sodium Fast Reactor [8] and fusion benchmarks [17]. In this study, the steps presented on the right part of Fig. 1 are used. We emphasize again that automation and a disciplined, quality assured working method (with emphasis on reproducibility) is imperative to accomplish this. First of all, the codes TALYS, NJOY and MCNP need to be very robust and secured against relatively large variations in input parameters. Next, all detailed knowledge about the material/benchmark in question should be present in the input files of these codes. It is clear that manual intervention must be completely excluded in the sequence of code calculations. Once all that is assured, the rest is relatively simple: if we can do a full calculation loop once, we can also do it 1000 times.

The input files for this method are a MCNP geometry input file (same as for the perturbation method) and $n$ random ENDF files (consistent with the unique ENDF file plus covariances used for the perturbation method). Each random ENDF file is produced by the TALYS code, is fully reproducible and consists of a unique set of nuclear data. Each random file is completely different from another one: nu-bar and energy released per fission (" $M F 1$ " in ENDF language), resonance parameters (" $M F 2$ "), cross sections ("MF3"), angular distributions ("MF4"), fission neutron spectrum ("MF5") and double differential data ("MF6") are varied.

For each random ENDF file, the processing by NJOY (to produce ACE files) and the benchmark calculation is performed with MCNP. At the end of the $n$ calculations, $n$ different $\mathrm{k}_{\mathrm{eff}}$ values with their statistical uncertainties are obtained. From the calculated probability distribution of $\mathrm{k}_{\mathrm{eff}}$, the standard deviation $\sigma_{\text {total }}$ reflects two different effects: $\sigma_{\text {total }}^{2}=\sigma_{\text {statistics }}^{2}+\sigma_{\text {nuclear data }}^{2}$. The first one $\left(\sigma_{\text {statistics }}\right)$ is from the statistical uncertainty derived from the number of histories (neutrons) used in the MCNP calculations. It typically varies as $1 / \sqrt{N}, N$ being the number of considered histories, is known in advance and in principle can be minimized by investing enough computer time. The second origin 
Table 1. Details of the comparison TMC-Perturbation method for four $\mathrm{k}_{\mathrm{eff}}$ benchmarks.

\begin{tabular}{|c|c|c|c|c|c|c|c|c|c|}
\hline & hst3 & $-6{ }^{19} \mathrm{~F}$ & $\mathrm{hmf}$ & $34{ }^{19} \mathrm{~F}$ & & $\mathrm{pmf}$ & ${ }^{239} \mathrm{Pu}$ & $\mathrm{pm}$ & ${ }^{240} \mathrm{Pu}$ \\
\hline & $\Delta \mathrm{k}_{\mathrm{e}}$ & $(\mathrm{pcm})$ & $\Delta \mathrm{k}_{\mathrm{e}}$ & $(\mathrm{pcm})$ & & $\Delta \mathrm{k}_{\mathrm{e}}$ & $(\mathrm{pcm})$ & $\Delta \mathrm{k}_{\mathrm{e}}$ & $(\mathrm{pcm})$ \\
\hline & TMC & $\begin{array}{l}\text { Pertur- } \\
\text { bation }\end{array}$ & TMC & $\begin{array}{l}\text { Pertur- } \\
\text { bation }\end{array}$ & & TMC & $\begin{array}{l}\text { Pertur- } \\
\text { bation }\end{array}$ & TMC & $\begin{array}{l}\text { Pertur - } \\
\text { bation }\end{array}$ \\
\hline Total & 333 & 287 & 346 & 286 & Total & 844 & 722 & 790 & 650 \\
\hline MF2 & 278 & 238 & 314 & 277 & MF1 & 400 & - & 370 & - \\
\hline MF3 & 172 & 161 & 75 & 105 & $(\mathrm{n}, \mathrm{inl})$ & 170 & 140 & 70 & 50 \\
\hline MF4 & 101 & - & 80 & - & $(\mathrm{n}, \mathrm{el})$ & 250 & 240 & 30 & 40 \\
\hline MF6 & 30 & - & 35 & - & $(\mathrm{n}, \gamma)$ & 100 & 100 & 30 & 30 \\
\hline & & & & & $(\mathrm{n}, \mathrm{f})$ & 720 & 660 & 730 & 640 \\
\hline & & & & & MF4 & 20 & - & 20 & - \\
\hline & & & & & MF5 & 50 & - & 30 & - \\
\hline & & & & & MF6 & 50 & - & 30 & - \\
\hline
\end{tabular}

( $\left.\sigma_{\text {nuclear data }}\right)$ lies in the use of different random nuclear data files (ACE files) between calculations. It induces a spread in the $\mathrm{k}_{\text {eff }}$ distribution, which can unequivocally be assigned to the spread of cross sections, angular distributions and so on. This spread is not known and is to be derived from the present Monte Carlo approach. The quadratic sum of the two distinct spreads is equal to the total observed standard deviation. If the observed spread is of the order of the statistical uncertainty (first effect), only a maximum value can be attributed to the spread due to nuclear data.

As mentioned previously, the TMC method allows to vary much more information than included in the covariance files used by the perturbation method, which considers resonance parameters ("MF2") and cross sections ("MF3") covariances. It seems then natural to always obtain a larger nuclear data uncertainty from the TMC method than from the perturbation method. In order to disentangle the contribution of each so-called $M F$ and reactions, additional ENDF random files are produced together with the full random ENDF files. In these additional files, only part of them are varied. For instance, MF2 random files are created were only resonance parameters are varied and the rest of the file stays constant, MF3 random files are created were only cross sections are varied, MF4 random files are created were only angular distributions are varied. In this manner, benchmarks can be calculated using these partially randomized files and the contribution of specific quantities can be obtained.

\section{Results}

After the necessary preliminary tests of consistency and convergence, comparisons of benchmarks uncertainties can be performed. In the following, a few benchmarks and isotopes with high sensitivity are selected. A direct comparison between the TMC and perturbation methods are presented in Table 1.

In general, the total uncertainties obtained with the TMC method are larger than with the perturbation method. For the studied benchmarks, the ratio of "TMC over Perturbation" is larger than 1. This can be easily foreseen because the complete nuclear data file is randomized in TMC (not only cross sections) compared to the four (major) cross sections considered with the perturbation method.

For the presented benchmarks, differences from 15 to $30 \%$ exist. Table 1 presents details of the uncertainties calculations for four benchmarks. The contributions of the four main cross sections directly come from SUSD for the perturbation method and in the case of the TMC method, separate calculations are done, with each time, randomizing only part of the nuclear data file. In ENDF terminology, $M F 2$ stands for the resonance range, $M F 3$ for cross sections in the fast neutron range, $M F 4$ for the elastic angular distribution, $M F 5$ for the fission neutron spectrum, $M F 6$ for the double differential data and MF1 for the $v$-bar and other fission quantities (such as released energy ...).

It can be seen in Table 1 that the contributions of MF1 and MF4 are not negligible. MF5 and MF6 have a smaller effect on $\mathrm{k}_{\mathrm{eff}}$. Additionally, in the case of cross sections where both methods provide results, TMC uncertainties are larger than the one from the perturbation approach. 
A possible explanation is that the information contained in the covariance files (used in the perturbation method) does not exactly match the random files (used in TMC). The difference can be related to the values of cross sections, cross section uncertainties, or probability distributions (Gaussian for covariance files and not Gaussian from the random files). More work is then necessary to study these differences. Alternatively, different energy groups (more than 33) can also be used to verify the impact of cross sections, uncertainties and sensitivities collapsing.

\section{Conclusion}

In the present work, a first attempt to compare two uncertainty propagation methods is presented. The first method, called TMC, provides a more general and exact answer, does not require special codes, but is more time consuming. The second method, called Perturbation method, considers a restricted number of nuclear data uncertainties, relies on perturbation theory, needs more processing and intermediate codes, but is the fastest to produce results. Preliminary comparisons show that nuclear data quantities not handled by the perturbation method are of importance (such $v$-bar or angular distributions), but the differences between the two methods ranges within 15 to $30 \%$.

In the future, the TMC method might become easier to use in any offices because of cheaper and more spread computer power. But for the time being, the perturbation method is still dominating the "market". An interesting question is: for how long ?

\section{References}

1. M.B. Chadwick et al., Nuclear Data Sheets 107, 2931(2006).

2. A.J. Koning and D. Rochman, "TENDL-2009: Consistent Talys-based Evaluated Nuclear Data Library including covariances", OECD/NEA JEF/DOC-1310, November 2009, available at http://www.talys.eu/tendl-2009.

3. A.J. Koning and D. Rochman, "Modern nuclear data evaluation: Straight from nuclear physics to applications", these proceedings.

4. "Uncertainty and target accuracy assessment for innovative systems using recent covariance data evaluations", report by the Working Party on International Evaluation Co-operation of the NEA Nuclear Science Committee, Volume 26, NEA/WPEC-26, 2008.

5. A. Hogenbirk, "An easy way to carry out 3D uncertainty analysis", Proceedings of the Joint International Topical Meeting on Mathematics and Computation and Supercomputing in $\mathrm{Nu}-$ clear Applications (M\&C +SNA 2007), Montery, California, April 15-19, 2007, on CD-ROM, American Nuclear Society, LaGrange Park, Il.

6. A.J. Koning and D. Rochman, Annals of Nuclear Energy 35, 2024(2008).

7. D. Rochman, A.J. Koning and S.C. van der Marck, Annals of Nuclear Energy 36, 810(2009).

8. D. Rochman, A.J. Koning, D.F. da Cruz, P. Archier and J. Tommasi, Nucl. Inst. and Methods A 612, 374(2010).

9. Briggs, J.B. Ed., 2004, International Handbook of evaluated Criticality Safety Benchmark Experiments, NEA/NSC/DOC(95)03/I, Organisation for Economic Co-operation and Development, Nuclear Energy Agency.

10. S.C. van der Marck, Nucl. Data Sheets 107, 3061(2006).

11. "MCNP - A general monte Carlo code n-particle transport code", version 4C, Report LA-13709M, Los Alamos National Laboratory, J.F. Briesmeister (Ed.), April 2000.

12. R.E. McFarlane and D.W. Miur, “The NJOY Nuclear Data Processing System, version 91”, Report LA-17740-M, Los Alamos National Laboratory, 1994.

13. R.E. McFarlane, "NJOY99 - code system for producing pointwise and multigroup neutron and photon cross sections from ENDF/B data", 2000, Los Alamos National Laboratory, RSIC PSR-480.

14. D. Wiarda, G. Arbanas, L. Leal and M.E. Dunn, Nucl. Data Sheets 109, 2791(2008). 
EFNUDAT Workshop “Measurements and Models of Nuclear Reactions", Paris, 2010

15. K. Furuta, Y. Oka and S. Kondo, "SUSD: A computer code for cross section sensitivity and uncertainty analysis including secondary neutron energy and angular distributions", report UTNLR0185, Nucl. Eng. Res. Lab, University of Tokyo, 1986, NEA Data Bank package n. 1151.

16. A.J. Koning, S. Hilaire and M.C. Duijvestijn, "TALYS-1.0", in the proceedings of the International Conference on Nuclear Data for Science and Technology- ND2007, May 22-27, 2007, Nice, France; www.talys.eu.

17. D. Rochman and A.J. Koning, "Exact nuclear data uncertainty propagation for fusion design", accepted in Fusion Engineering and Design, September 2009. 